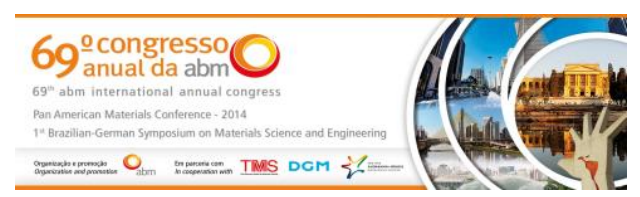

Tema: Corrosão

\title{
ESTUDO DO COMPORTAMENTO DA RESISTÊNCIA À CORROSÃO DA LIGA CO-W OBTIDA PELO PROCESSO DE ELETRODEPOSIÇÃO USANDO TARTARATO DE SÓDIO COMO AGENTE COMPLEXANTE*
}

\author{
Anamélia de Medeiros Dantas \\ Mislene Pereira Lins ${ }^{2}$ \\ José Eudes Lima Santos ${ }^{1}$ \\ José Leonardo Costa Raulino ${ }^{3}$ \\ Aureliano Xavier dos Santos ${ }^{4}$ \\ Theophilo Moura Macie/ ${ }^{5}$ \\ Shiva Prasad ${ }^{6}$ \\ Renato Alexandre Costa de Santana \\ Ana Regina Nascimento Campos ${ }^{8}$
}

\section{Resumo}

Os revestimentos protetores, além de constituírem uma importante forma de prevenção à corrosão metálica, principalmente a atmosférica pode melhorar consideravelmente as propriedades físico-químicas. A eletrodeposição representa um método alternativo para revestimentos de superfície metálica e tem a finalidade de inibir a corrosão, que ocorre na superfície do metal sob a influência do meio ambiente. A liga de Co-W tem gerado considerável interesse devido a sua resistência ao desgaste e a corrosão. No processo de eletrodeposição do tungstênio não podem ser reduzidos completamente ao estado de valência zero em meio aquoso, mas é possível depositá-los na presença dos elementos do grupo do ferro. O presente trabalho propõe avaliar a influência do agente complexante tartarato de sódio no processo de obtenção da liga Co-W. Como ferramenta de otimização foi utilizado à metodologia de superfície de resposta. As medidas eletroquímicas de corrosão foram executadas em uma célula convencional de três eletrodos. Foram utilizadas as mediadas de polarização potenciodinâmicos linear. Foi avaliada a composição química das ligas pelo EDX e a morfologia da superfície com o MEV. O melhor resultado de eletrodeposição para a liga Co-W foi obtido com concentrações sulfato de cobalto $0.2 \mathrm{M}$, tartarato de sódio $0.3 \mathrm{M}$ e tungstato de sódio $0.05 \mathrm{M}$ e apresentou morfologia nodular com conteúdos de tungstênio de $38 \%$ e cobalto de $62 \%$ e uma boa resistência a corrosão.

Palavras-chave: Liga Co-W; Corrosão; Eletrodeposição; Tartarato de sódio.

\section{Abstract}

STUDY ON CORROSION RESISTENCE BEHAVIOR OF CO-W ALLOY OBTAINED BY THE ELECTRODEPOSITION PROCESS USING SODIUM TARTARATE AS COMPLEXING AGENT

Protective coatings, besides serving as an important way to prevent the metalic corrosion, particularly air corrosion, can greatly improve the physico-chemical properties. Electrodeposition is an alternative method for coating a metal surface and is intended to inhibit the corrosion that occurs on the metal surface under the influence of the environment. Co-W alloy has generated considerable interest due to its corrosion and wear resistance. In the process of electrodeposition, tungsten can not be completely reduced to zero valence state in aqueous medium, but it can be deposited in presence of the iron group elements. This study aims to evaluate the influence of complexing agent sodium tartrate in the process of obtaining the Co-W alloy. Response surface methodology was used as an optimization tool. The electrochemical corrosion measurements were performed in a conventional three-electrode cell. The linear potentiodynamic polarization measurements were used for corrosion characterization. The chemical composition of the alloy was evaluated by EDX and surface morphology by SEM. The best result for the electrodeposition of Co-W alloy was obtained with $0.2 \mathrm{M}$ cobalt sulfate, $0.3 \mathrm{M}$ sodium tartrate and $0.05 \mathrm{M}$ sodium tungstate and showed nodular morphology with $38 \%$ tungsten and $62 \%$ cobalt and a good corrosion resistance.

Keywords: Co-W alloy; Corrosion; Electrodeposition; Sodium tartarate.

1 Graduando, Licenciatura em Química, bolsista PIBIC, Universidade Federal de Campina Grande (UFCG), Cuité, PB, Brasil.

2 Química, Universidade Estadual da Paraíba, Técnica em Laboratório de Química, Centro de Educação e Saúde, Unidade Acadêmica de Educação, UFCG, Mestranda, Ciências Naturais e Biotecnologia, UFCG, Cuité, PB, Brasil.

3 Graduando em Licenciatura em Química, Universidade Federal de Campina Grande, Cuité, PB, Brasil

4 Engenheiro de Materiais, Doutorando em Ciências e Engenharias dos Materiais, UFCG, Campina Grande, PB, Brasil.

5 Engenheiro Mecânico, Dr., Prof., Unidade Acadêmica de Engenharia Mecânica, UFCG, Campina Grande, PB, Brasil.

6 Químico, Doutor, Pesquisador, Unidade Acadêmica de Educação, UFCG, Cuité, PB, Brasil.

7 Químico, Doutor, Professor, Unidade Acadêmica de Educação, UFCG, Cuité, PB, Brasil.

8 Químico Industrial, Doutora, Professora, Unidade Acadêmica de Educação, UFCG, Cuité, PB, Brasil.

\footnotetext{
* Contribuição técnica ao 69ำ Congresso Anual da ABM - Internacional e ao 14ํㅡㄹ ENEMET - Encontro Nacional de Estudantes de Engenharia Metalúrgica, de Materiais e de Minas, 21 a 25 de julho de 2014, São Paulo, SP, Brasil.
} 


\section{INTRODUÇÃO}

Até pouco tempo, o termo corrosão era utilizado para descrever um determinado tipo de deterioração dos metais, não se aplicando a materiais não metálicos [1]. Entretanto, o termo "corrosão" atualmente, pode ser definido como a reação do metal com os elementos do seu meio, no qual o metal é transformado a um estado de óxido [2]. Quando este processo ocorre o metal perde suas propriedades essenciais, tais como durabilidade, resistência mecânica, elasticidade, ductilidade e o produto de corrosão formado é extremamente pobre destas propriedades. A corrosão metálica é causada pela ação química ou eletroquímica do meio, a qual pode estar ou não associada, a uma ação física [3]. Com o avanço tecnológico, mundialmente alcançado, e consequente aumento da demanda de produção, os custos da corrosão evidentemente se elevam, tornando-se um fator de grande importância a ser considerado já na fase de projeto de grandes instalações industriais para evitar ou minimizar futuros processos corrosivos. Por isso faz-se necessário o desenvolvimento de materiais que visem combater ou amenizar 0 efeito da corrosão.

Os revestimentos protetores, além de constituírem uma importante forma de prevenção à corrosão metálica, principalmente a atmosférica pode melhorar consideravelmente as propriedades físico-químicas [4]. A eletrodeposição representa um método alternativo para revestimentos de superfície metálica e tem a finalidade de inibir a corrosão, que ocorre na superfície do metal sob a influência do meio ambiente.

A eletrodeposição é um método que consiste em depositar uma camada protetora fina de liga metálica sobre a superfície de um substrato [5]. Entre os revestimentos mais comuns estão os de cobre, níquel e cromo [6]. As ligas eletrodepositadas exibem propriedades superiores quando comparadas àquelas dos elementos constituintes.

A liga de Co-W tem gerado considerável interesse devido as suas propriedades magnéticas, resistência à corrosão e ao desgaste. De acordo com Bairachna [7] na eletrodeposição do tungstênio e molibdênio, não podem ser reduzidos completamente há um estado de valência zero em eletrólitos aquosos, mas é possível depositá-lo como uma liga com o ferro, cobalto e níquel.

Agentes complexantes têm sido amplamente utilizados em eletrodeposição de ligas para aumentar a estabilidade do banho. $\mathrm{Na}$ eletrodeposição da liga Co-W foi utilizado como agente complexante o tartarato de sódio, que é um quelante com dois grupos doadores. O complexante presente no banho eletrolítico comporta-se como uma base de Lewis enquanto que os íons dos metais $\left(\mathrm{Co}^{2+}, \mathrm{W}^{6+}\right)$ compartam-se como ácido de Lewis [8].

O planejamento fatorial experimental apresenta muitas vantagens sobre os métodos univariantes. Neste método os fatores são simultaneamente variados ao mesmo tempo e não um-a-um como nos métodos clássicos, permitindo observar as interações antagônicas e a sinergia envolvida nos fatores analisados. A Metodologia de Superfície de Resposta (MSR) é uma coleção de técnicas matemáticas e estatísticas usada para desenvolvimento, melhoramento e otimização de processos, e pode ser usada para avaliar a significância relativa de muitos fatores que são afetados por interações complexas. O objetivo principal da MSR é determinar as condições ótimas de operação para um sistema ou determinar uma região que satisfaça as especificações operacionais [9].

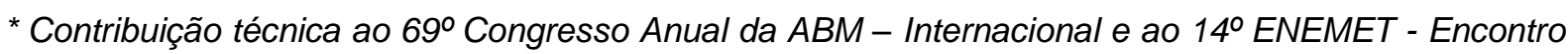
Nacional de Estudantes de Engenharia Metalúrgica, de Materiais e de Minas, 21 a 25 de julho de 2014, São Paulo, SP, Brasil.
} 


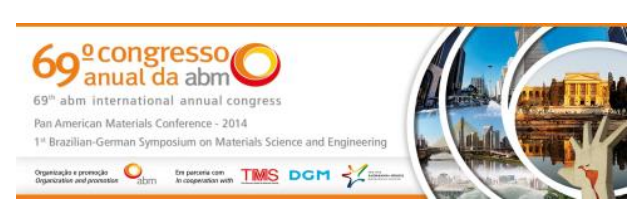

\section{MATERIAIS E MÉTODOS}

\subsection{Preparação do banho e substrato}

O banho eletroquímico foi preparado, utilizando reagentes com alto grau de pureza analítica e com água deionizada. O banho eletroquímico utilizado na eletrodeposição da liga de Co-W foi constituído dos reagentes descritos na Tabela 1. O pH do banho foi ajustado adicionando-se hidróxido de amônio $\left(\mathrm{NH}_{4} \mathrm{OH}\right)$ e/ou ácido sulfúrico $\left(\mathrm{H}_{2} \mathrm{SO}_{4}\right)$. O substrato utilizado foi uma placa de cobre com área superficial de $8 \mathrm{~cm}^{2}$; esta foi inicialmente polida com lixa de 400, 600 e 1200 mesh e, em seguida, foi feito o tratamento químico, mergulhando o substrato em soluções de $10 \%$ de $\mathrm{NaOH}$ para remover algum álcali residual e de $1 \%$ de $\mathrm{H}_{2} \mathrm{SO}_{4}$ para ativar a superfície.

Tabela 1. Valores reais e codificados das variáveis independentes (composição do banho).

\begin{tabular}{lccc}
\hline \multicolumn{1}{c}{ Níveis } & -1 & 0 & 1 \\
Reagentes & & & $0.2 \mathrm{M}$ \\
Sulfato de cobalto & $0.05 \mathrm{M}$ & $0.125 \mathrm{M}$ & $0.3 \mathrm{M}$ \\
Tungstato de sódio & $0.05 \mathrm{M}$ & $0.175 \mathrm{M}$ & $0.7 \mathrm{M}$ \\
Tartarato de sódio & $0.3 \mathrm{M}$ & $0.5 \mathrm{M}$ & \\
\hline
\end{tabular}

\subsection{Eletrodeposição}

A eletrodeposição foi realizada sobre o substrato quadrado de cobre, operando como o catodo, que foi inserido no interior de um eletrodo cilíndrico de platina (anodo). Um potenciostato/galvanostato MQPG-01 da MICRO QUIMICA foi utilizado no controle da densidade de corrente catódica. Um eletrodo rotatório EG\&G PARC 616 foi utilizado no controle de rotação catódica.

As eletrodeposições foram realizadas usando os seguintes parâmetros, densidade de corrente $40 \mathrm{~mA} / \mathrm{cm}^{2}$, agitação mecânica 30rpm, carga 500 Coulomb, a temperatura do banho foi mantida em $60^{\circ} \mathrm{C} \pm 2^{\circ} \mathrm{C}$ e $\mathrm{pH} 8,0$.

\subsection{Ensaios de corrosão}

As medidas eletroquímicas de corrosão foram realizadas em uma célula convencional de três eletrodos. Foram utilizadas as medidas de polarização potenciodinâmica linear (PPL). Nessas medidas foi utilizado 0 potenciostato/galvanostato PG STAT 30 da AUTOLAB conectado ao software Nova. $O$ eletrodo de trabalho foi o substrato de cobre revestido com a liga de Co-W. $O$ eletrodo de referencia utilizado foi calomelano saturado (ECS), o contra eletrodo foi um fio de platina em espiral. Todos os teste de corrosão eletroquímica foram realizados em meio corrosivo contendo 0,1 Molar de $\mathrm{NaCl}$ em solução de temperatura ambiente (aproximadamente $25^{\circ} \mathrm{C}$ ) e com uma taxa de varredura de $1 \mathrm{mV} \mathrm{s}^{-1}$.

\subsection{Composição e Morfologia}

A composição e morfologia do revestimento foram realizadas utilizando um EDX (espectrômetro de fluorescência de Raios-X por energia dispersiva, SHIMADZU EDX-720) para a determinação da composição do revestimento metálico e a espessura do mesmo. Foi também realizado um ensaio de MEV (Microscopia

\footnotetext{
* Contribuição técnica ao $69^{\circ}$ Congresso Anual da ABM - Internacional e ao 14ํㅡㄹ ENEMET - Encontro Nacional de Estudantes de Engenharia Metalúrgica, de Materiais e de Minas, 21 a 25 de julho de 2014, São Paulo, SP, Brasil.
} 


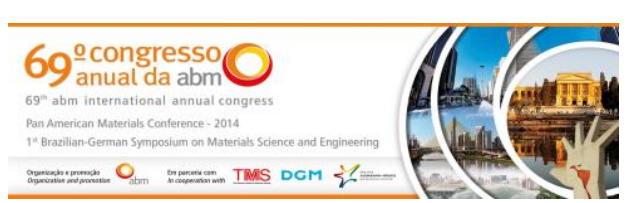

Eletrônica de Varredura, SHIMADZU, modelo X550). Com este ensaio pode se observar a morfologia dos depósitos obtidos, quanto ao surgimento de trincas e nódulos.

\subsection{Planejamento Fatorial}

$\mathrm{Na}$ analise experimental foi utilizada a metodologia de superfície de resposta (MSR) para analisar a influência das variáveis independentes nas variáveis de respostas. $O$ comportamento do sistema foi representado utilizando um planejamento fatorial de $2^{3}$. Esse planejamento é utilizado quando se quer observar como a resposta (resistência à corrosão) dependerá das interações dos três fatores (concentração de sulfato de cobalto, tungstato de sódio e tartarato de sódio).

A analise de variância (ANOVA) é uma ferramenta para comparação de varias variável [10]. Com a ANOVA é possível investigar diferenças significativas entre as variáveis dependentes.

\section{RESULTADOS E DISCUSSÃO}

Os resultados de resistência à corrosão (potencial de corrosão, resistência de polarização) foram tratados com 0 auxilio da matriz fatorial $2^{3}$ utilizada para a otimização dos componentes do banho eletrolítico da liga Co-W e são mostradas na Tabela 2. Foram realizados 10 experimentos sendo 2 pontos centrais, esses experimentos foram realizados em duplicatas para confirmar a sua reprodutibilidade, totalizando 20 experimentos.

Tabela 2. Variáveis, níveis, valores de potencial de corrosão, resistência de polarização para o planejamento fatorial $2^{3}$.

\begin{tabular}{cccccc}
\hline Experimento & $\begin{array}{c}\text { Sulfato de } \\
\text { cobalto }\end{array}$ & $\begin{array}{c}\text { Tungstato de } \\
\text { sódio }\end{array}$ & $\begin{array}{c}\text { Tartarato de } \\
\text { sódio }\end{array}$ & $E_{\text {corr }}(\mathrm{V})$ & $R_{p}(\mathrm{Ohm})$ \\
\hline 1 & $-1(0.05)$ & $-1(0.05)$ & $-1(0.3)$ & -0.792 & 1627.4 \\
2 & $1(0.2)$ & $-1(0.05)$ & $-1(0.3)$ & -0.785 & 4738.3 \\
3 & $-1(0.05)$ & $1(0.3)$ & $-1(0.3)$ & -0.782 & 859.09 \\
4 & $1(0.2)$ & $1(0.3)$ & $-1(0.3)$ & -0.789 & 913.71 \\
5 & $-1(0.05)$ & $-1(0.05)$ & $1(0.7)$ & -0.79 & 1571.9 \\
6 & $1(0.2)$ & $-1(0.05)$ & $1(0.7)$ & -0.792 & 1269.5 \\
7 & $-1(0.05)$ & $1(0.3)$ & $1(0.7)$ & -0.809 & 731.53 \\
8 & $1(0.2)$ & $1(0.3)$ & $1(0.7)$ & -0.828 & 535.71 \\
$9(\mathrm{C})$ & $0(0.125)$ & $0(0.175)$ & $0(0.5)$ & -0.799 & 1071.9 \\
$10(\mathrm{C})$ & $0(0.125)$ & $0(0.175)$ & $0(0.5)$ & -0.79 & 906.93 \\
1 & $-1(0.05)$ & $-1(0.05)$ & $-1(0.3)$ & -0.77 & 1813.3 \\
2 & $1(0.2)$ & $-1(0.05)$ & $-1(0.3)$ & -0.75 & 5325.6 \\
3 & $-1(0.05)$ & $1(0.3)$ & $-1(0.3)$ & -0.77 & 992.54 \\
4 & $1(0.2)$ & $1(0.3)$ & $-1(0.3)$ & -0.74 & 2016.3 \\
5 & $-1(0.05)$ & $-1(0.05)$ & $1(0.7)$ & -0.79 & 1383.4 \\
6 & $1(0.2)$ & $-1(0.05)$ & $1(0.7)$ & -0.79 & 1526.4 \\
7 & $-1(0.05)$ & $1(0.3)$ & $1(0.7)$ & -0.87 & 996.33 \\
8 & $1(0.2)$ & $1(0.3)$ & $1(0.7)$ & -0.847 & 1374.4 \\
$9(\mathrm{C})$ & $0(0.125)$ & $0(0.175)$ & $0(0.5)$ & -0.84 & 897.82 \\
$10(\mathrm{C})$ & $0(0.125)$ & $0(0.175)$ & $0(0.5)$ & -0.83 & 694.07 \\
\hline
\end{tabular}

Os resultados foram submetidos a uma analise de regressão não linear múltipla para obterem-se os graus de cada parâmetro. Estimativas dos coeficientes com níveis mais altos que $95 \%(P<0,05)$ foram incluídas no modelo final [4]. A resistência de

\footnotetext{
* Contribuição técnica ao $69^{\circ}$ Congresso Anual da ABM - Internacional e ao 14ํㅡㄹ ENEMET - Encontro Nacional de Estudantes de Engenharia Metalúrgica, de Materiais e de Minas, 21 a 25 de julho de 2014, São Paulo, SP, Brasil.
} 
polarização $\left(R_{p}\right)$ pode ser representada na forma de função dos fatores independentes por um modelo matemático de $1^{\text {a }}$ ordem.

A analise de variância (ANOVA) dos dados demonstrou a significância estatística do modelo, justificando assim o uso do modelo de $1^{\text {a }}$ ordem para o estudo estatístico. A avaliação estatística foi determinada pelo teste de Fisher para analise de variância, Tabela 3, e apresentou um $\mathrm{R}^{2}$ igual 0.88 .

Tabela 3. Resultado da ANOVA para resistência a corrosão.

\begin{tabular}{cccccc}
\hline Fontes & Soma quadrática & $\begin{array}{c}\text { Grau de } \\
\text { liberdade }\end{array}$ & Média quadrática & $F$ & $P$ \\
\hline (1)Sulfato de cobalto & 3729176 & 1 & 3729176 & 12.57798 & 0.004022 \\
(2)Tungstato de sódio & 7338938 & 1 & 7338938 & 24.75319 & 0.000322 \\
(3)Tartarato de sódio & 4947366 & 1 & 4947366 & 16.68675 & 0.001513 \\
Interação entre 1 e 2 & 1692061 & 1 & 1692061 & 5.70708 & 0.034201 \\
Interação entre 1 e 3 & 3685181 & 1 & 3685181 & 12.42959 & 0.004180 \\
Interação entre 2 e 3 & 2730533 & 1 & 2730533 & 9.20970 & 0.010375 \\
Interação entre 1, 2 e 3 & 2165658 & 1 & 2165658 & 7.30445 & 0.019214 \\
Erro residual & 3557815 & 12 & 296485 & & \\
Soma total & 29846729 & 19 & & & \\
\hline
\end{tabular}

\subsection{Efeito do tartarato de sódio}

Para eletrodepositar a liga de Co-W deve-se adicionar um agente complexante, como ex., o tartarato de sódio. A concentração apropriada do tartarato de sódio no banho melhora a solubilidade dos íons metálicos proporcionando um depósito com maior aderência.

O efeito da concentração do tartarato de sódio foi avaliado em um intervalo de 0,3 a $0,7 \mathrm{M}$. A analise de regressão dos dados experimentais na faixa de estudo mostrou que a concentração foi uma variável significativa no processo de eletrodeposição, para um nível de confiança de 95\% (Equação 1).

$R_{p}=1562+482.78^{\star} A-556^{\star} B-325^{\star} C-479.9^{\star} A^{\star} B+413^{\star} B^{\star} C+367.9^{\star} A^{\star} B^{\star} C-33.863$ Equação 1

Onde, (A) é a concentração do sulfato de cobalto, (B) é a concentração de Tartarato de sódio, $(C)$ é a concentração do tungstato de sódio, $(A B)$ é a interação entre as concentrações de sulfato de cobalto e tartarato de sódio, (ABC) é a interação entre as concentrações de sulfato de cobalto, tungstato de sódio e tartarato de sódio.

A Figura (Figura 1) mostra o comportamento do tartarato vs. à concentração de sulfato de cobalto. À medida que diminui a concentração do tartarato de sódio e aumenta à concentração do sulfato de cobalto o melhor resultado de resistência a corrosão é obtido. Isso ocorre devido a menor concentração do complexante permite que os íons fiquem mais livres, ou seja, com o aumento da concentração do tartarato aumenta também a solubilidade dos metais (tornam-se mais estáveis), diminuindo assim a quantidade de íons reduzidos no sistema. A Figura 2 mostra que com a maior concentração de sulfato de cobalto e menor concentração de tartarato se obteve a menor corrente de corrosão.

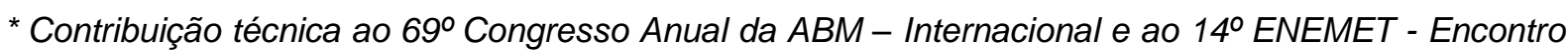
Nacional de Estudantes de Engenharia Metalúrgica, de Materiais e de Minas, 21 a 25 de julho de 2014, São Paulo, SP, Brasil.
} 


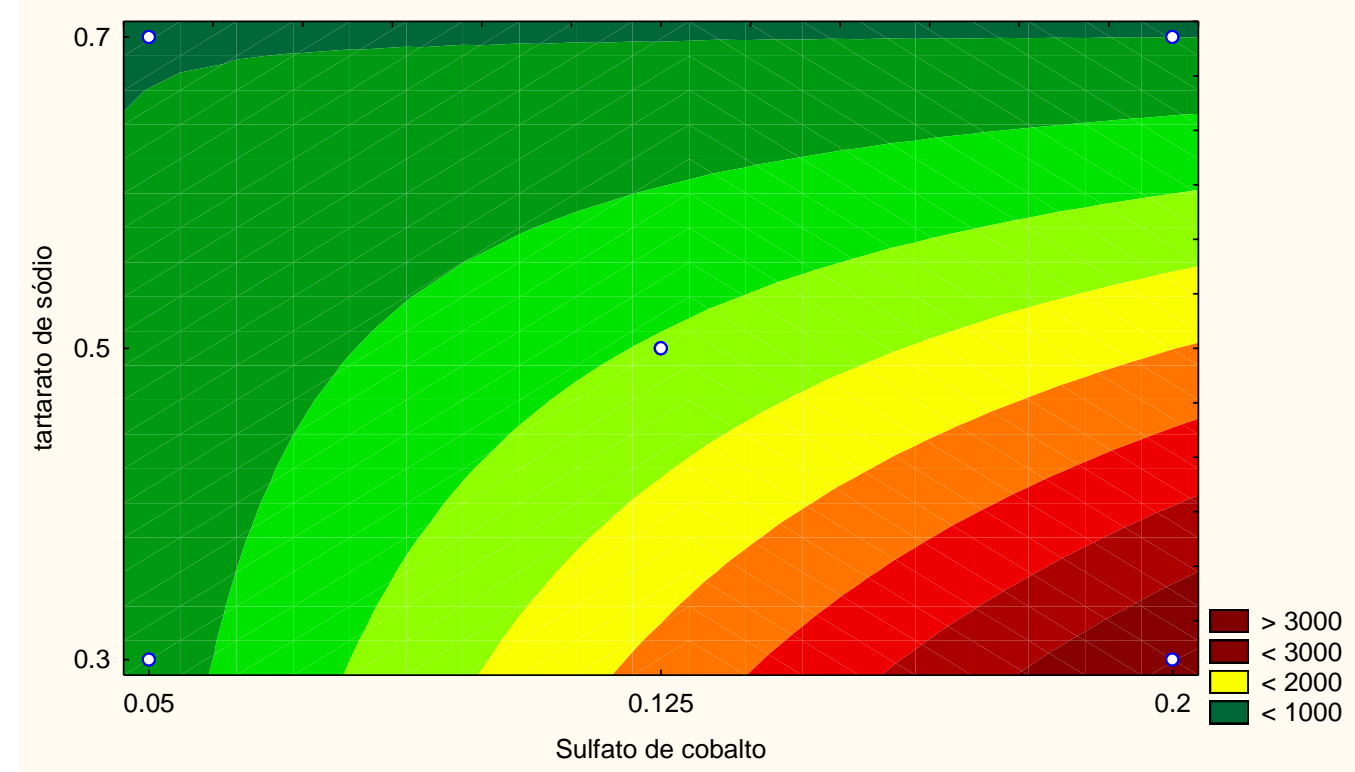

Figura 1. Superfície de resposta da resistência à corrosão sob a influência da concentração de tartarato vs. sulfato de cobalto, usando $0.05 \mathrm{M}$ de tungstato de sódio.

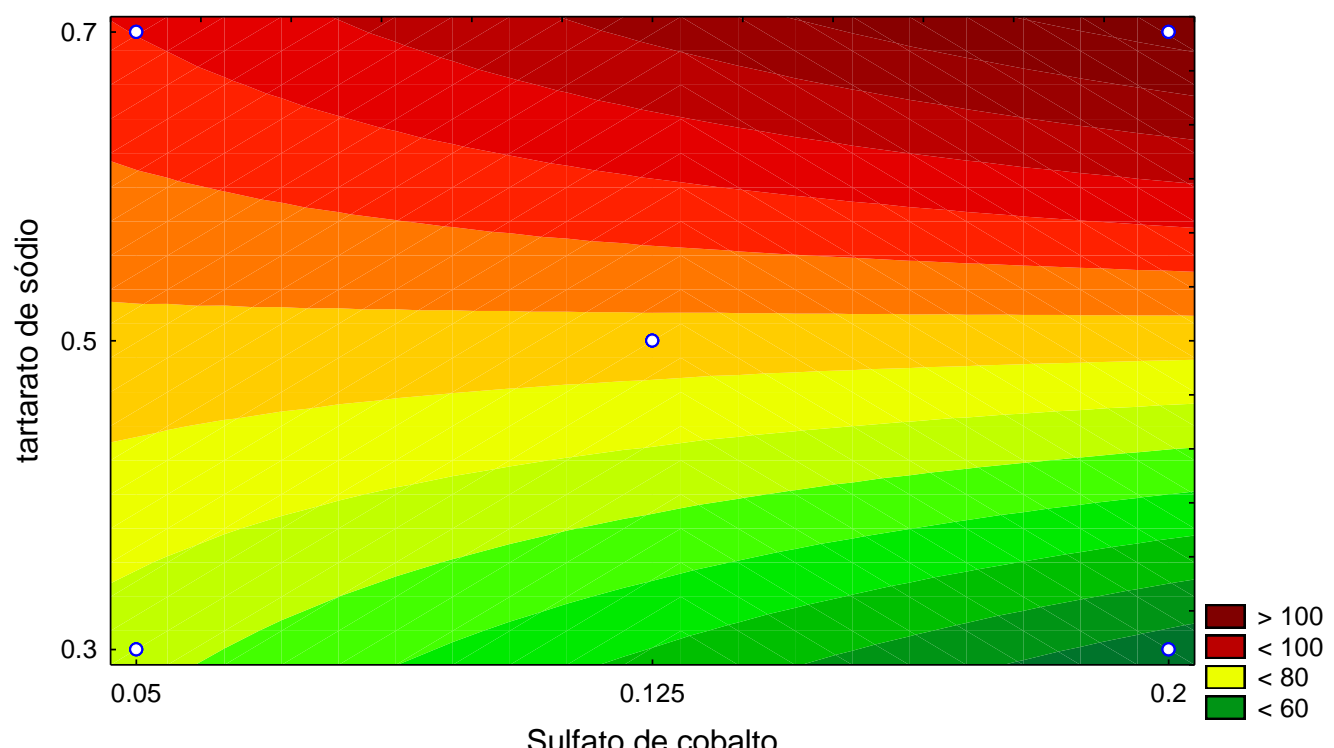

Figura 2. Superfície de resposta da corrente de corrosão sob a influência da concentração de tartarato vs. sulfato de cobalto, usando $0.05 \mathrm{M}$ de tungstato de sódio.

\subsection{Efeito do sulfato de cobalto}

O estudo do efeito da concentração do sulfato de cobalto foi avaliado em um intervalo de 0,05 a $0,2 \mathrm{M}$. A Figura 3 mostra o comportamento do efeito da concentração do sulfato de cobalto vs. tungstato de sódio. À medida que aumenta a concentração do sulfato de cobalto aumenta a composição de tungstênio no revestimento como mostra a Tabela 4 , isso pode ocorre devido a eletrodeposição ser induzida, ou seja, o cobalto serve como um indutor para que ocorra a deposição do tungstênio. Podlaha e Landolt [11], propuseram um mecanismo de deposição da liga Ni-Mo que sugere que o íon do metal do grupo do ferro proporcione a redução do elemento induzido (molibdênio e tungstênio) através da formação de um óxido intermediário adsorvido na superfície do eletrodo.

\footnotetext{
* Contribuição técnica ao 69ำ Congresso Anual da ABM - Internacional e ao 14ํㅡㄹ ENEMET - Encontro Nacional de Estudantes de Engenharia Metalúrgica, de Materiais e de Minas, 21 a 25 de julho de 2014, São Paulo, SP, Brasil.
} 
A Figura 4 mostra a relação das concentrações de sulfato de cobalto e tungstato de sódio sobre a corrente de corrosão. À medida que a concentração de sulfato de cobalto aumenta e a de tungstato de sódio diminui, são obtidas as menores correntes de corrosão.

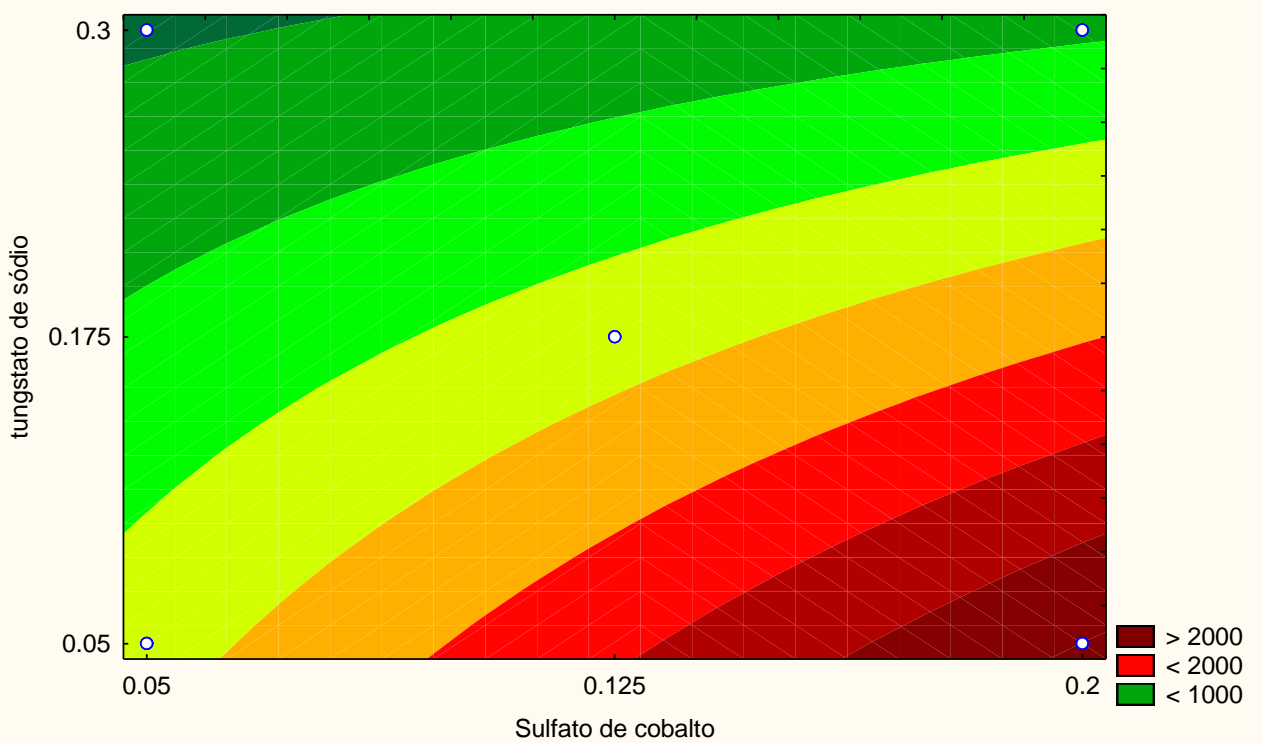

Figura 3. Superfície de resposta da resistência à corrosão sob a influência da concentração do tungstato de sódio vs. sulfato de cobalto, usando $0.3 \mathrm{M}$ de tartarato de sódio.

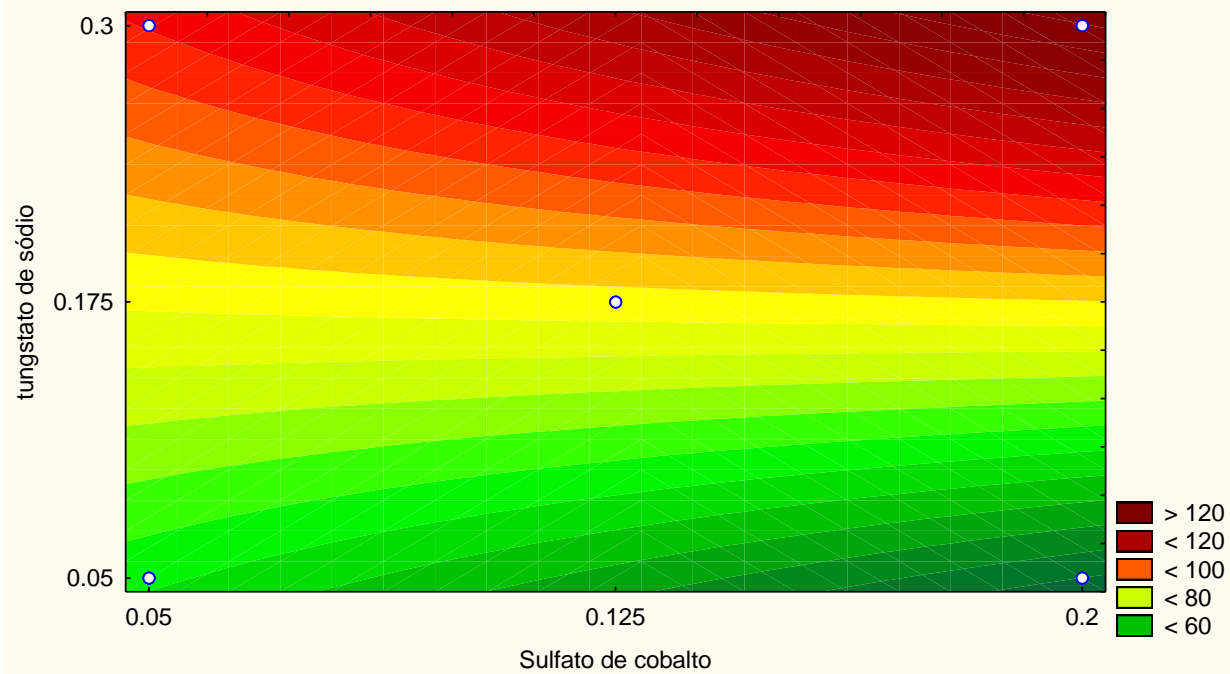

Figura 4. Superfície de resposta da corrente de corrosão sob a influência da concentração do tungstato de sódio vs. sulfato de cobalto, usando $0.3 \mathrm{M}$ de tartarato de sódio.

Tabela 4. Composição química da liga de Co-W após obtidos no EDX.

\begin{tabular}{cccccc}
\hline Experimento & Sulfato de cobalto & Tungstato de sódio & Tartarato de sódio & Co (wt\%) & W (wt\%) \\
\hline $\mathbf{1}$ & $-1(0.05)$ & $-1(0.05)$ & $-1(0.3)$ & 91,38 & 8,62 \\
$\mathbf{2}$ & $1(0.2)$ & $-1(0.05)$ & $-1(0.3)$ & 62,39 & 37,61 \\
$\mathbf{3}$ & $-1(0.05)$ & $1(0.3)$ & $-1(0.3)$ & 84,94 & 15,06 \\
$\mathbf{4}$ & $1(0.2)$ & $1(0.3)$ & $-1(0.3)$ & 64,30 & 35,70 \\
$\mathbf{5}$ & $-1(0.05)$ & $-1(0.05)$ & $1(0.7)$ & 89,07 & 10,93 \\
$\mathbf{6}$ & $1(0.2)$ & $-1(0.05)$ & $1(0.7)$ & 70,77 & 29,23 \\
$\mathbf{7}$ & $-1(0.05)$ & $1(0.3)$ & $1(0.7)$ & 73,05 & 26,95 \\
$\mathbf{8}$ & $1(0.2)$ & $1(0.3)$ & $1(0.7)$ & 65,72 & 34,28 \\
$\mathbf{9}$ & $0(0.125)$ & $0(0.175)$ & $0(0.5)$ & 85 & 15 \\
$\mathbf{1 0}$ & $0(0.125)$ & $0(0.175)$ & $0(0.5)$ & 83 & 17 \\
\hline
\end{tabular}

\footnotetext{
* Contribuição técnica ao 69ํ Congresso Anual da ABM - Internacional e ao 14ํㅡㄹ ENEMET - Encontro Nacional de Estudantes de Engenharia Metalúrgica, de Materiais e de Minas, 21 a 25 de julho de 2014, São Paulo, SP, Brasil.
} 


\subsection{Composição e aparência do deposito}

Os resultados de EDX exibidos na Tabela (Tabela 4) mostram que a uma tendência sobre a concentração do sulfato de cobalto, ou seja, com uma maior concentração de sulfato de cobalto nota-se o aumento no teor de tungstênio (wt\%) e uma diminuição no teor de cobalto na liga.

As Figuras (Figura 5a e Figura 5b) mostram a analise do MEV correspondente aos revestimentos da liga de Co-W. A superfície do melhor revestimento em relação à resistência à corrosão Figura (Figura $5 \mathrm{a}$ ) apresentou a formação de nódulos que tem uma morfologia quase esférica, o mesmo foi observado por Ghaferi et al. [12]. Já na Figura (Figura 5b) há o surgimento de trincas isso pode ter sido ocasionado pela liberação de hidrogênio na eletrodeposição e pela tensão interna provocada pelo cobalto na liga. Segundo Mizushima et al. [13], o ingresso de hidrogênio para o depósito ocorre durante a eletrodeposição e sua posterior liberação cria um estresse de alta resistência, que pode desenvolver as microfissuras no depósito.

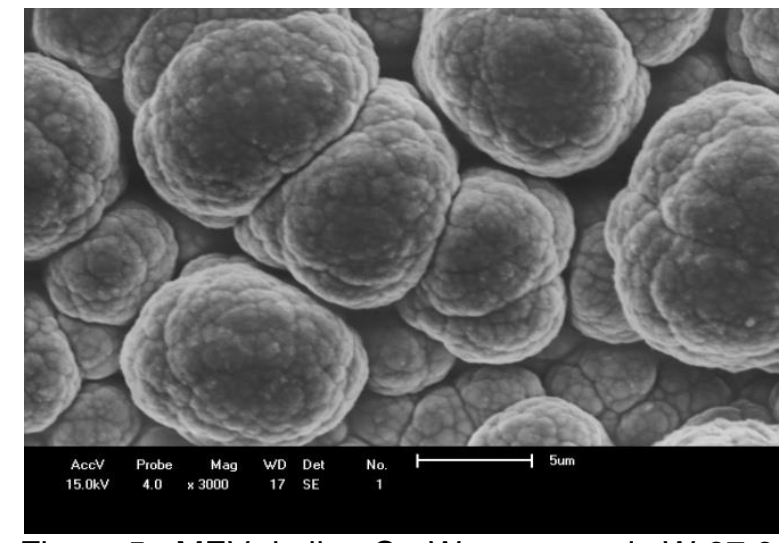

Figura 5a MEV da liga Co-W com teor de W 37,61 wt\%.

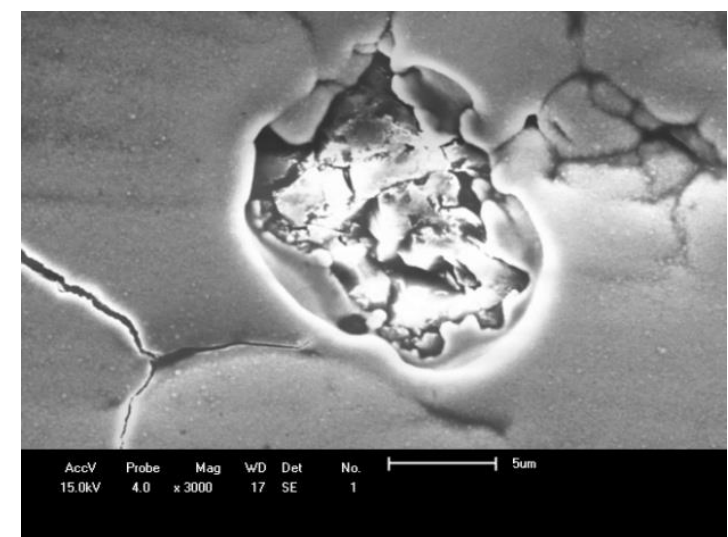

Figura 1b MEV da liga Co-W com teor de W 10.93 wt\%.

\section{CONCLUSÃO}

A partir da análise do planejamento fatorial $2^{3}$ pode-se constatar o melhor resultado de eletrodeposição da liga de Co-W com concentração de sulfato de cobalto $0.2 \mathrm{M}$, concentração de tungstato de sódio $0.05 \mathrm{M}$ e concentração de tartarato de sódio 0.3M. O tratamento estatístico demonstrou uma interação de primeira ordem para os valores das concentrações, para a obtenção do melhor resultado.

A liga binária de Co-W apresentou o surgimento nódulos com morfologia quase esférica. A composição química do melhor resultado apresentou um teor de tungstênio 38 wt\% e um teor de cobalto 62 wt\%.

\section{Agradecimentos}

Ao CNPq pelo financiamento do projeto e pela concessão da bolsa PIBIC; Ao professor Renato Alexandre Costa de Santana pela orientação.

\section{REFERÊNCIAS}

1 Uhlig HH. Corrosion and control. New York : John Wiley \& Sons; 1962.

2 Ponte HA. Fundamentos de corrosão. Depart. Eng. Química. 2003

3 Gentil V. Corrosão. Ed.5a. Rio de Janeiro: LTC; 2007.

\footnotetext{
* Contribuição técnica ao 69ำ Congresso Anual da ABM - Internacional e ao 14ํㅡㄹ ENEMET - Encontro Nacional de Estudantes de Engenharia Metalúrgica, de Materiais e de Minas, 21 a 25 de julho de 2014, São Paulo, SP, Brasil.
} 


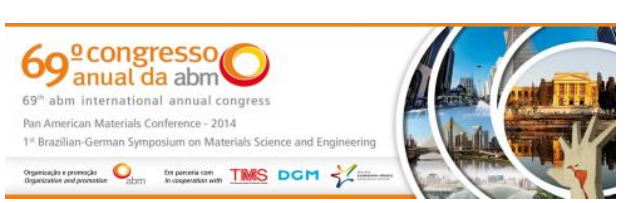

4 Santana RAC, Campos ARN, Prasad S. Otimização do banho eletrolítico da liga Fe-W-B resistente à corrosão. Quim. Nova. 2007; 30 (2):360-365.

5 Santana RAC, Prasad S, Santana FSM. Revestimentos eletrolítico com uma liga amorfa de Ni-W-B, resistente à corrosão e ao desgaste. Eclética Química. 2003; 28 (1): 69-72

6 Hamid ZA. Electrodeposition of cobalt-tungsten alloys from acidic bath containing cationic surfactants. Materials Letters. 2003; 57 (16-17): 2558-2564.

7 Bairachna T. Electrodeposition of Ni-W, Ni-W-Mo and Ni-Mo alloys thin films and Ni-W nanowires [master thesis]. Massachusetts: Northeastern University Boston; 2011.

8 Melo LC, Lima Neto P, Correia AN. The influence of citrate and tartrate on the electrodeposition and surface morphology of Cu-Ni layers. J. Appl. Electrochem. 2011; 41: 415-422.

9 Ravikumar K, Krishnan S, Ramalingam S, Balu K. Optimization of process variables by the application of response surface methodology for dye removal using a novel adsorbent. Dyes and Pigments. 2007; 72: 66-74.

10 Paese C, Caten CT, Ribeiro JLD. Aplicação da ánalise de variância na implantação do CEP. Revista Produção. 2001; 11 (01).

11 Podlaha HA, Landolt JD. Induced Codeposition: iii. Molybdenum alloys with nickel, cobalt and iron. Electrochem. Soc. 1996; 143 (3): 885.

12 Ghaferi Z, Raeissi K, Golozar MA, Saatchi A, Kabi S. Comparison of electrodeposition aspects and characteristics of $\mathrm{Ni}-\mathrm{W}$ and $\mathrm{Co}-\mathrm{W}$ alloy nanocrystalline coatings. Iranian Journal of Materials Science \& Engineerin. 2010; 7(4).

13 Mizushima I, Tang PT, Hansen NH, Somers MAJ. Development of a new electroplating process for Ni-W alloy deposits. Electrochim. Acta, 2005; 51: 888-896.

* Contribuição técnica ao 69 Congresso Anual da ABM - Internacional e ao 14ํㅡㄹ ENEMET - Encontro Nacional de Estudantes de Engenharia Metalúrgica, de Materiais e de Minas, 21 a 25 de julho de 2014, São Paulo, SP, Brasil. 